Service social

\title{
Travail social et participation parentale dans le contexte du partenariat école-famille-communauté : mise en perspective autour d'une logique socioculturelle
}

\author{
Dany Boulanger, François Larose, Serge J. Larivée, Pauline Minier, Yves \\ Couturier, Jean-Claude Kalubi-Lukusa et Valérie Cusson
}

Volume 57, numéro 2, 2011

URI : https://id.erudit.org/iderudit/1006294ar

DOI : https://doi.org/10.7202/1006294ar

Aller au sommaire du numéro

Éditeur(s)

École de service social de l’Université Laval

ISSN

1708-1734 (numérique)

Découvrir la revue

Citer cet article

Boulanger, D., Larose, F., Larivée, S. J., Minier, P., Couturier, Y., Kalubi-Lukusa, J.-C. \& Cusson, V. (2011). Travail social et participation parentale dans le contexte du partenariat école-famille-communauté : mise en perspective autour d'une logique socioculturelle. Service social, 57(2), 74-95.

https://doi.org/10.7202/1006294ar

\section{Résumé de l'article}

L'exercice de la profession du travail social évolue dans le contexte de la mise en oeuvre d'un partenariat entre l'école, la famille et la communauté. Le travailleur social s'y voit attribuer un rôle de soutien à la participation des parents dans l'univers scolaire. Pour plusieurs considérations, les modalités de définition et les conditions d'exercice d'un tel rôle font contraste avec ce qui caractérise son agir professionnel, en particulier dans le champ familial. Après avoir mis en tension les deux logiques d'action, nous ferons appel à une perspective socioculturelle pour circonscrire le rôle travailleur social au regard d'une fonction de médiation culturelle. 


\title{
Travail social et participation parentale dans le contexte du partenariat école-famille-communauté : mise en perspective autour d'une logique socioculturelle
}

\author{
Dany Boulanger \\ François Larose \\ Serge J. Larivée \\ Pauline Minier \\ Yves Couturier \\ Jean-Claude Kalubi-Lukusa \\ Valérie Cusson
}

\begin{abstract}
RÉSUMÉ
L'exercice de la profession du travail social évolue dans le contexte de la mise en œuvre d'un partenariat entre l'école, la famille et la communauté. Le travailleur social s'y voit attribuer un rôle de soutien à la participation des parents dans l'univers scolaire. Pour plusieurs considérations, les modalités de définition et les conditions d'exercice d'un tel rôle font contraste avec ce qui caractérise son agir professionnel, en particulier dans le champ familial. Après avoir mis en tension les deux logiques d'action, nous ferons appel à une perspective socioculturelle pour circonscrire le rôle travailleur social au regard d'une fonction de médiation culturelle.
\end{abstract}

Mots clés : Participation parentale; partenariat école-famille-communauté; pratique professionnelle; travail social; culture.

\begin{abstract}
The social work profession operates in the context of the implementation of a partnership between school, family and community. In this context, the social worker is assigned the role of supporting parent participation in the school environment. This role is put into perspective regarding the practices relating to the participation and social action that are characteristic of the profession. The tensions that arise between the fields of Education and Social work lead us to define this role around a socio-cultural logic where the Social worker is considered a cultural mediator.
\end{abstract}

Key Words: Parental participation, school, family, and community partnership; professional practices; social work, culture. 


\section{INTRODUCTION}

Cet article se situe dans le contexte de l'émergence, au Québec, d'un discours entourant l'établissement d'un partenariat entre l'école, la famille et la communauté. La mise en œuvre de projets, de mesures, de programmes ou, plus largement, de pratiques à caractère partenarial passe par la mobilisation des professionnels du milieu, tels que le travailleur social, dans l'univers scolaire. Le travailleur social est appelé à favoriser et à soutenir la participation parentale. Considérant que la participation sociale des parents est à la base de son agir professionnel, nous mettrons en tension le sens donné à la participation parentale dans le champ du partenariat école-famille-communauté et dans le domaine du travail social. En nous appuyant sur une conception socioculturelle de ce construit, nous mettrons en perspective le rôle qu'est appelé à exercer le travailleur social en matière de soutien à la participation parentale dans le cadre de pratiques dites partenariales. Les limites soulevées à cet égard nous conduiront à proposer une définition de ce rôle fondée sur une finalité de médiation culturelle (i.e., médiation entre les cultures de la famille, de l'école et de la communauté).

\section{Le rôle du travailleur social à la charnière entre l'école, la famille et la communauté : le contexte actuel}

Un discours en faveur de l'établissement d'un partenariat entre l'école, la famille et la communauté est en émergence au Québec. Les intervenants du milieu, tels que le travailleur social, sont appelés à franchir les portes de l'école. Ils sont alors de plus en plus concernés par la chose scolaire. L'action éducative ne relève plus du seul ressort de l'enseignant, comme c'était le cas jadis (Proulx, 2009). Au contraire, les discours actuels font de la réussite éducative une responsabilité collective partagée (Department of Education, 2006, 2007; Gouvernement du Québec, 2009a, 2009b).

Dans le cadre de la réforme actuelle du curriculum scolaire, amorcée officiellement avec la création de la nouvelle Loi sur l'instruction publique en 1998, plusieurs changements structurels ont été effectués en faveur d'une articulation entre l'action éducative et socioéducative des divers intervenants au cœur et à la charnière de l'espace scolaire (Gouvernement du Québec, 2000; Larose, Lenoir, Couturier et Bédard, 2011sp). De même, le ministère de la Santé et des Services sociaux a convenu avec celui de l'Éducation, du Loisir et du Sport d'une série de mesures de collaboration intersectorielle, dont le texte fondateur est une entente cadre (Gouvernement du Québec, 2003a). Une lecture comparative des principaux documents ministériels produits respectivement par ces ministères au cours des dix dernières années permet de constater que la réussite éducative de l'élève est de plus en plus évoquée comme cible d'intervention des intervenants du milieu, dont, au premier chef, le travailleur social $\left(2003 b, 2006 a, 2006 b, 2007,2008^{1}\right)$. Un tel exercice met aussi en évidence le fait que la préoccupation pour une action globale et écosystémique exercée sur l'enfant et sur sa famille, dimension qui caractérise l'agir professionnel du travailleur social, caractérise la documentation officielle en éducation (Gouvernement du Québec, 2000, 2002a, 2002b, 2003c, 2003d, 2005).

1. Le programme national de santé publique (2003-2012), publié à l'origine en 2003, a été mis à jour en 2008 et présenté dans une seconde version. Celle-ci intègre la dimension de réussite éducative de manière plus manifeste que dans le premier document produit. 
Nous assistons alors à une forme d'enchevêtrement des discours qui témoigne d'une volonté d'articuler l'action éducative et socioéducative des intervenants du milieu à celle des professionnels œuvrant au sein de l'institution scolaire. Dans cette logique, la pratique du travailleur social est appelée à s'inscrire foncièrement dans le champ de l'intervention éducative et socioéducative (Larose, Lenoir, Couturier et Bédard, 2011sp).

L'intervention éducative renvoie à l'appropriation contextualisée de savoirs par l'enfant et passe par l'action de médiation d'un ensemble de professionnels de métiers relationnels tels que l'enseignant et le travailleur social (Couturier et Larose, 2006; Lenoir, Larose, Deaudelin, Kalubi et Roy, 2002). L'intervention est socioéducative si elle comporte une dimension éducative, si elle est structurée et contextualisée, si elle est intégratrice des compétences d'un ensemble d'acteurs et si elle soutient les apprentissages tant formels qu'informels réalisés par l'enfant, et ce, dans, autour ou hors de l'espace scolaire (Larose, Lenoir, Couturier et Bédard, 2011sp; Terrisse et Larose, 2003; Terrisse, Larose et Couturier, 2003).

Au sein de l'équipe-école, notion floue désignant l'ensemble des acteurs œuvrant au sein de l'institution scolaire ou soutenant ses actions éducatives, le personnel enseignant collabore avec les professionnels des services complémentaires. Ces derniers sont définis par un ensemble d'activités, d'ordre varié, visant à supporter l'enfant dans différentes sphères de son développement et à faciliter ses apprentissages dans une logique de continuité avec l'exercice des pratiques enseignantes. L'articulation de l'action de l'ensemble des personnels scolaires autour de cibles communes (les compétences transversales et les domaines généraux de formation) serait une condition facilitant cette cohérence de la pratique éducative et socioéducative (Gouvernement du Québec, 2002a, 2005). Ils collaborent directement au sein de rencontres d'équipe ou indirectement par le biais de cet arrimage entre leurs actions respectives. La collaboration, souvent employée comme un synonyme de partenariat, est ici entendue au sens d'un processus par lequel les intervenants co-définissent un ensemble de pratiques et de savoirs dans le but d'atteindre des cibles ou des objectifs communs. La nature, le degré et l'intensité de ce processus varient considérablement selon les conditions de la pratique et l'option épistémologique sous-jacente (Vincent et Tomlinson, 1997).

Au sein de l'école, les intervenants éducatifs comme l'enseignant et les intervenants socioéducatifs, tels que les psychoéducateurs et les travailleurs sociaux, partagent des objectifs; ils visent le soutien, le transfert et la contextualisation des apprentissages de l'enfant (Gouvernement du Québec, 2000, 2002a). Le personnel des services complémentaires ne fait pas que soutenir l'enseignant lorsque les enfants présentent des problèmes de conduite. II favorise l'appropriation des apprentissages par l'élève et leur transfert à des situations quotidiennes significatives par une action qui porte sur certains des déterminants psychologiques, sociaux ou de santé de l'apprentissage. De leur côté, les enseignants ne ciblent pas que la dimension académique, mais soutiennent par exemple le sentiment de 
citoyenneté chez l'élève ${ }^{2}$ (Couturier, Potvin, Lortie, Etheridge, Lantagne, Lacasse-Bédard et Bédard, 2010).

Les personnels scolaires collaborent ensemble notamment dans le cadre de la définition, de la mise en œuvre et de l'évaluation des plans de réussite. Ces derniers constituent un cadre de référence commun à l'intervention éducative et socioéducative. II permet d'opérationnaliser la mission éducative de l'école et ses grandes orientations sous la forme d'objectifs. Ces derniers sont foncièrement associés à la réussite éducative de l'élève et sont soutenus par des moyens concrets identifiés explicitement dans le plan.

Le plan de réussite est le produit d'une collaboration entre les membres du personnel de l'école, mais aussi de ceux-ci avec des parents et des membres et représentants du milieu communautaire. II est en effet défini au sein des conseils d'établissement de l'école, une structure participative composée, de manière paritaire, de représentants des parents, des personnels scolaires, des citoyens ainsi que de la direction d'école. La responsabilité première des conseils d'établissement consiste à favoriser le partenariat entre l'école, la famille et la communauté, particulièrement en mobilisant les acteurs du milieu autour de la mission éducative de l'école et la participation des parents (Gouvernement du Québec, 2002a). En ce sens, le plan de réussite comporte une forte dimension partenariale; en particulier, la participation parentale y figure souvent comme une composante clé.

Bien que, dans les faits, le leadership en matière de définition et de mise en œuvre des plans de réussite soit particulièrement exercé par les enseignants et les directions d'école (Deniger et Roy, 2002), les parents et les représentants des organismes du milieu sont de plus en plus actifs au sein des conseils d'établissement, en raison de l'évolution des politiques scolaires en faveur d'une reconnaissance du rôle partenarial des parents et des acteurs du milieu (Proulx, 2009). Par ailleurs, ils jouent un rôle mobilisateur accru par leur participation active au sein de groupes d'intérêts qui exercent, en périphérie de l'école, une influence marquée sur les politiques et les pratiques scolaires. Souvent ces groupes comportent des dispositifs de mobilisation, des structures décisionnelles rattachées à l'école, comme c'est le cas de la Fédération des comités de parents.

À travers les conseils d'établissement, mais aussi dans le cadre de programmes, de mesures ou de projets à caractère partenarial dont le plan de réussite constitue parfois l'élément rassembleur (ex. : École en santé, Stratégie d'intervention Agir autrement, Famille, école, communauté : réussir ensemble, etc.), les intervenants socioéducatifs du milieu, tels que les travailleurs sociaux, sont appelés à articuler leur action éducative et socioéducative à celle des personnels scolaires (Gouvernement du Québec, 2005). Ils y collaborent plus particulièrement à la définition et à la mise en œuvre de moyens visant à promouvoir la participation parentale dans l'univers scolaire.

2. Pour des informations plus détaillées sur l'articulation prescrite de leurs actions éducatives et socioéducatives, consultez le guide Les services éducatifs complémentaires : essentiels à la réussite (Gouvernement du Québec, 2002). 


\section{La participation parentale : éléments de définition}

Dans le domaine du partenariat école-famille-communauté, la participation parentale est un construit polysémique qui ne comporte pas de base de définition solide et dont l'acception varie considérablement d'un auteur à un autre (Christenson, 2004; Jordan, Orozco et Averett, 2001). Il est souvent employé comme un synonyme de construits apparentés tels que l'implication ou l'engagement et même confondu avec les notions de partenariat et de collaboration qui sont plus englobantes (Cairney, 2000; Fan et Chen, 2001).

La participation des parents est généralement circonscrite autour des «activités » dans lesquelles ils s'impliquent et qui comportent une forte composante scolaire (Christenson, 2004). Les auteurs renvoient alors à l'implication ou à la participation « scolaire » des parents (Lupiani, 2004). Les écrits scientifiques font référence le plus souvent aux activités définies et mises en place par les intervenants éducatifs et socioéducatifs dans le cadre de mesures et de programmes à caractère partenarial (Epstein, 1994, 2005; Simon, 2004). II ne s'agit pas des activités auxquelles participent les parents dans leur quotidien et qu'ils initient, mais de celles fixées et déterminées par les personnels scolaires (Christenson et Sheridan, 2001).

La définition des activités en question varie considérablement d'un auteur à un autre. En effet, il existe une multitude de typologies de l'«implication scolaire des parents », où la participation est inscrite dans des formes multiples et souvent peu apparentées au modèle auquel les auteurs font référence. Certains auteurs classent les activités selon le degré de mobilisation parentale auxquelles elles font appel (Clénet et Gérard, 1994; Henderson et Mapp, 2002; Larivée, Kalubi et Terrisse, 2006; Powell, 2001). Ainsi, par exemple, la participation scolaire des parents en tant que bénévoles à l'école est considérée comme une activité de nature moins partenariale que ne l'est leur implication dans le processus décisionnel de l'école. Certains auteurs mentionnent que c'est la logique épistémologique ${ }^{3}$ autour de laquelle s'articule la notion de participation parentale et qui façonne les pratiques éducatives et socioéducatives qui permet le mieux de traduire le sens donné à ce construit et la façon dont cette participation se traduit et s'opérationnalise (Boethel, 2003; Lawson, 2003; Souto-Manning et Swick, 2006; Vincent et Tomlinson, 1997). La présence parentale à un comité décisionnel ne garantit pas sa participation optimale. L'exercice d'un rôle d'agent de soutien de seconde ligne ou de technicien ou encore de partenaire ${ }^{4}$ de la part du parent dépend de la conception de la participation véhiculée en recherche et adoptée par l'institution scolaire et les professionnels se mobilisant dans le cadre d'un projet à caractère partenarial (Lawson, 2003; Souto-Manning et Swick, 2006).

3. Ici, nous entendons l'épistémologie au sens d'une métathéorie ou d'un métadiscours, une forme de filet rassembleur qui donne une orientation particulière à un construit et aux pratiques qui lui sont associées et qui articule entre elles les composantes de ces pratiques (Overton, 1998). Il s'agit alors de la « conception » d'une pratique véhiculée en recherche et traduite par l'adoption d'une « lecture » particulière de l'intervention par les praticiens. Cette lecture réfère plus particulièrement aux croyances des professionnels ou à leurs représentations sociales du parent et de sa participation (Boulanger, Larose et Couturier, 2010). Nous renvoyons le lecteur à Shommer-Aikins (2004) pour saisir l'incidence de l'«épistémologie » du chercheur et des professionnels sur la pratique.

4. De manière générique, un partenaire est un acteur qui partage ses compétences, qui co-construit un ensemble de savoirs et de pratiques avec des tiers dans le cadre d'un processus de collaboration basé sur une interaction égalitaire et réciproque (Christenson et Sheridan, 2001). 


\section{Les conceptions dominantes de la participation parentale}

Dans le champ du partenariat école-famille-communauté, deux conceptions de la participation parentale dominent et elles servent de référence aux pratiques dites partenariales. II s'agit d'une lecture déficitaire et d'une vision scolaire de la participation parentale (Lawson, 2003; Souto-Manning et Swick, 2006). Boethel (2003) y ajoute une perspective interactionniste qu'elle associe à un paradigme scolaire.

Dans une perspective déficitaire, la participation parentale se définit par l'exercice d'un rôle marginal de la part des parents, en particulier ceux issus d'un milieu socio-économiquement faible (msef) dont les caractéristiques sont considérées comme des contraintes potentielles aux apprentissages de l'enfant (Lawson, 2003). Les intervenants résistent généralement à la présence parentale à l'école, comme ils leur attribuent une forme d'incompétence en matière de soutien aux apprentissages de l'élève (Boudreault, Kalubi et Moreau, 2000; Boudreault, Kalubi, Sorel, Beaupré et Bouchard, 1998; Van Velsor et Orozco, 2007). La participation parentale se restreint alors généralement à la sphère familiale, les parents soutenant la réalisation des travaux scolaires à la maison (Nichols, 2007). Ils ont aussi pour rôle de créer un environnement éducatif familial stimulant pour l'enfant dans le but de préparer ce dernier à son entrée à l'école et de soutenir son cheminement scolaire.

Dans une perspective scolaire, la participation parentale se traduit dans des formes d'activités plus actives au sens où les parents y sont davantage mobilisés (Lawson, 2003; Souto-Manning et Swick, 2006). Ces derniers sont invités à s'impliquer à l'école. Ils peuvent assister à des événements collectifs, tels que la rentrée scolaire, ou participer à leur organisation, en tant que bénévoles. Parfois ils assistent l'enseignant en classe ou encore participent au processus de prise de décisions de l'école, comme membres du conseil d'établissement par exemple. Ils sont aussi invités à communiquer avec les personnels scolaires.

Bien que leur participation soit prononcée, ils exercent rarement un rôle de partenaire, c'està-dire qu'ils n'interagissent pas sur une base de réciprocité et de mutualité avec les professionnels et qu'ils ne partagent pas et ne co-construisent pas de compétences avec eux (Christenson et Sheridan, 2001; Moll, 1993). S'ils s'impliquent dans le processus décisionnel de l'école, ils exercent souvent une faible influence sur les politiques et les pratiques éducatives, leurs points de vue étant généralement peu pris en considération (Christenson, 2004). Au sein des conseils d'établissement de l'école qui, à la base, sont censés constituer une structure de mobilisation active des parents, considérés comme des partenaires, ces derniers exercent un faible leadership et ils se disent généralement insatisfaits du rôle qu'ils y jouent (Proulx, 2009).

La communication entre eux et les intervenants est souvent unidirectionnelle, ces derniers ayant la plupart du temps l'initiative du lien (Lott, 2001). Cette communication se restreint souvent à l'envoi d'informations de nature scolaire. Les professionnels contactent le plus souvent les parents lorsque l'enfant présente des difficultés d'adaptation scolaire ou sociale ou qu'il manifeste un risque d'en développer (Eldridge, 2001). Les parents sont invités à participer à des activités pour «faire à la maison comme on fait à l'école », c'est-à-dire pour soutenir le processus de transmission de savoirs scolaires à l'enfant, pour appuyer la mission de l'école et 
pour renforcer les pratiques éducatives exercées par les personnels scolaires, en particulier les pratiques pédagogiques des enseignants (Christenson, 2004; Powell, 2001).

Plusieurs auteurs, qui s'associent aux travaux d'Epstein (1995, 2005), adoptent une conception interactionniste de la participation parentale (Deslandes et Bertrand, 2004; Deslandes et Lemieux, 2005; Sanders et Lewis, 2004; Sanders et Simon, 2002; Sheldon, 2003, 2005). Ils proposent de fonder cette dernière sur l'interaction entre l'école, la famille et la communauté. Dans cette perspective, la participation scolaire des parents est considérée comme une condition majeure de rapprochement entre ces trois systèmes. Elle faciliterait alors la continuité des apprentissages que réalise l'enfant lorsqu'il passe d'un milieu ou d'un système à un autre.

L'articulation entre les systèmes dépend de la présence parentale à l'école. Dans cette logique, le fait, pour les parents, de franchir les portes de l'école, les expose aux valeurs, aux contenus curriculaires, aux savoirs transmis à l'enfant et aux pratiques éducatives qui y sont exercées (Lawson, 2003). Par le fait même, sa participation à l'école est censée l'amener à adhérer à la «culture » de l'école et, plus particulièrement, à favoriser la transmission de savoirs scolaires à l'enfant. Le parent est alors considéré comme une courroie de transmission d'informations scolaires (Samaras et Wilson, 1999).

Dans la perspective développée par Epstein, le lien entre l'école et la famille demeure essentiellement unidirectionnel et se situe dans une perspective hiérarchique ${ }^{5}$ (HooverDempsey et Sandlers, 1995; Price-Mitchell, 2009). Pour ces auteurs, c'est la responsabilité des parents d'aller vers l'école et non pas celle des intervenants de rechercher les compétences familiales et de s'appuyer sur ces dernières, par exemple en adaptant les pratiques pédagogiques aux caractéristiques des familles, notamment dans le contexte de l'apprentissage par projets. Comme dans une perspective scolaire, le parent adopte un rôle de réceptacle et de transmission d'informations et de savoirs scolaires (Boethel, 2003).

\section{La participation parentale dans une perspective socioculturelle}

Nous proposons d'évoquer une conception émergente de la participation parentale, fondée sur la prise en compte de la dimension de culture (Cairney, 2001; Gauvain, 2005; Pelligrini, Galda et Charak, 1997). Situer la participation dans une logique socioculturelle permet de reconnaître les systèmes sociaux comme des environnements socioculturels et ainsi de fonder les conduites des acteurs sur les repères normatifs qu'ils partagent en tant que membres d'un milieu (Cairney, 1997). En psychologie culturelle, la notion de culture est définie par les

5. Le modèle lui-même n'a bien sûr aucune incidence directe sur la pratique. Toutefois, en fonction des paramètres théoriques, conceptuels et épistémologiques qui le caractérisent, ce modèle organise l'action d'une façon particulière, un peu comme le fait une métathéorie (définie en note de bas de page ci-dessus). Cette analyse critique est lourde de sens et suppose de nombreuses nuances. Pour n'en rester qu'au plan théorique, c'est la conception de l'écosystème (de l'environnement social et du rapport entre les systèmes) ou de l'écosystémie (l'approche écosystémique elle-même) qui expliquerait la tendance des auteurs sollicitant le modèle d'Epstein à se situer dans une perspective scolaire. En effet, le rapport entre les systèmes y est peu défini, sinon dans une logique d'interaction minimale et unilatérale. À ce titre, nous renvoyons le lecteur à Price-Mitchell (2009) qui est, à notre connaissance, l'auteur qui construit le plus clairement cette analyse. En amont, nous proposons aussi au lecteur de consulter Larose, Terrisse, Lenoir et Bédard (2004). 
croyances, les savoirs et les pratiques constitutifs d'un milieu donné (Valsiner, 2009). Les croyances et les savoirs sont considérés comme des artéfacts, soit des objets de nature sociosymbolique qui déterminent l'action et les conduites sociales des individus (Roth, Tobin, Elmesky, Carambo, McKnight et Beers, 2004). Dans cette acception, les objets culturels s'apparentent à des représentations sociales, soit des savoirs de sens commun qui façonnent la participation sociale des individus ainsi que leurs pratiques et qui sont construits par le biais de leurs actions sociales (Valsiner, 2003a). La culture est foncièrement appréhendée dans une logique dynamique et processuelle, l'interaction entre les acteurs, leur participation sociale ainsi que leur appropriation de la culture étant constitutives de cette dernière (Valsiner, 2003b).

La culture est ainsi construite dans l'action, par le biais de la co-participation des individus à des pratiques et à des activités communes (Rogoff, 1997). La participation sociale favorise l'intégration des individus au sein d'un milieu et favorise la co-construction de savoirs et l'articulation entre les différents indices sémiotiques portés par chaque individu, en l'occurrence les attitudes ou les croyances (Bruni, Gherardi et Parolin, 2007). Dans cette logique, l'école est considérée comme un environnement socioculturel, et, à ce titre, les professionnels qui y interviennent partagent un certain nombre de repères sociosymboliques qui façonnent leurs actions éducatives et socioéducatives.

Dans le cadre de projets collaboratifs ou de nature partenariale, les acteurs appartenant à différents milieux co-construisent une communauté de pratiques, c'est-à-dire qu'ils élaborent conjointement un certain nombre de repères normatifs ou de savoirs de sens commun à la base des pratiques éducatives et socioéducatives qu'ils exercent auprès de l'enfant (Sing et Khine, 2006). Ils y adoptent une position de co-éducateurs et y réalisent des apprentissages par le biais de leurs interactions ainsi que de leur participation sociale.

\section{La participation parentale en travail social}

Traditionnellement, l'agir professionnel du travailleur social se circonscrit autour du « lien », de l'interaction et de la relation (Couturier, 2005). En intervention familiale, le travailleur social tend à faciliter la participation des parents. Il s'agit soit de l'engagement des parents dans le développement de l'enfant, soit de leur participation sociale au sein de la société, de la communauté ou d'institutions telles que l'école.

La participation sociale des parents est au cœur de la documentation scientifique en travail social dans les domaines du développement social ou communautaire, et dans celui de l'empowerment (Solas, 1996). Le discours sur la participation sociale en travail social s'articule foncièrement autour de l'intégration sociale des individus, de l'exercice de leur pensée critique et de leur pouvoir d'action, de leur appropriation de savoirs et de compétences ainsi que de la consolidation de leur identité sociale (Franklin et Sloper, 2005; Geoghegan et Powell, 2006; Roose, Mottart, Dejonckheere, Van Nijnatten et De Bie, 2009; Terrisse et Larose, 2003; Valtonen, 2002). Cette lecture concorde avec une conception socioculturelle de la participation parentale. 
Le parallèle peut être plus particulièrement établi autour des fondements de l'écosystémie sollicitée en travail social, soit, de la perspective de Lewin et ses collaborateurs (Lewin, 1935, 1943; Lewin et Grabbe, 1945; Lewin, Lippitt et White 1939) ainsi que de celle de Mead (1940, 1944, 1945a, 1945b, 1983, 2001) dont se revendique Bronfenbrenner $^{6}$ (1979), auteur qui constitue une figure de proue en travail social. Dans cette logique, le rapport qu'entretient l'individu avec sa réalité sociale dépend de son appartenance à une culture particulière et des interactions sociales qui façonnent notamment son identité et son statut social.

La participation sociale peut alors être considérée comme un moyen de construire et de s'approprier la culture. Les individus co-construisent un ensemble de repères culturels communs (des pratiques sociales de référence et des savoirs de sens commun) qu'ils s'approprient de manière contextualisée par le biais de leur participation au sein et à la charnière d'espaces socioculturels constitués de réseaux et de systèmes sociaux.

\section{Mise en perspective de la participation parentale en travail social autour du discours entourant le partenariat école-famille-communauté}

Le discours sur le partenariat école-famille-communauté est peu porteur d'une telle conception de la participation parentale. Le fait pour le parent d'être considéré comme externe à l'école ne permettrait pas au travailleur social d'exercer son rôle de soutien de liens sociaux. De façon générale, les professionnels des services complémentaires et du milieu favorisent la participation parentale sans considérer les systèmes école, famille et communauté comme des environnements socioculturels fondés sur des pratiques sociales et sur des repères socioculturels et normatifs (Delgado-Gaitan, 1992).

La notion de participation véhiculée dans le champ du partenariat école-famille-communauté ne se situe pas dans une logique socioculturelle. La participation parentale est le plus souvent définie dans une perspective a-culturelle, les auteurs ne prenant généralement pas en considération les fondements socioculturels des pratiques et de la participation parentale (Cairney, 2001).

Dans cette logique, les professionnels soutiennent peu la participation des parents à des projets et des activités communs construits au sein d'une communauté de pratique composée de l'ensemble des personnels scolaires et de leurs « partenaires ». Ils ne sont pas appréhendés comme des agents de construction de la culture scolaire ou, plus largement, d'une culture éducative. Par exemple, le curriculum de l'école est très généralement défini et implanté dans

6. L'écosystémie et, particulièrement, le modèle de Bronfenbrenner, sont souvent sollicités sans référence à leurs fondements théoriques et épistémologiques (Sontag, 1996). Par ailleurs, Bronfenbrenner lui-même tendrait à articuler son modèle de 1979 autour d'une conception «fonctionnelle » de l'environnement social et d'une lecture comportementaliste du rapport qu'entretient l'enfant avec son environnement, perspectives qui contredisent ses propres fondements (Henrich, 2006; Magnusson et Stattin, 1998; Overton, 1998; Tudge, Gray et Hogan, 1997; Wong, 2001). Le modèle bioécologique (Bronfenbrenner, 2005; Bronfenbrenner et Evans, 2000) qu'il développe ultérieurement semble s'inscrire en cohérence plus forte avec ces fondements. Le fait de nous appuyer sur ces derniers, soit sur les approches théoriques développées par Lewin et Mead, nous fournit un référent stable, qui nous permet d'effectuer des comparaisons entre deux champs (partenariat école-famillecommunauté versus une zone de pratique en travail social). Nous mettons alors en perspective la notion de participation, au cœur de l'écosystémie, à la lumière de ces deux champs. 
une logique descendante (top-down) et le leadership est généralement assumé par les enseignants et les directions d'école au plan de son actualisation. Ainsi, les parents n'ont généralement pas droit de parole au niveau des contenus qui y sont véhiculés et des pratiques exercées à l'école ou en périphérie de cette dernière. Le curriculum est alors foncièrement circonscrit autour de la culture de l'école et ses objets rejoignent très peu les besoins et plus largement la culture des familles de msef (Mills et Gale, 2007; Ogbu, 1992).

La participation parentale s'exerce plutôt en périphérie de l'école, à l'extérieur de l'environnement scolaire. Les professionnels véhiculent généralement des représentations sociales négatives à l'égard des compétences éducatives parentales et, parallèlement, ils reconnaissent peu les savoirs socioculturels et les pratiques sociales de référence qui caractérisent l'environnement familial (Boulanger, Larose et Couturier, 2010).

Articulant leurs pratiques autour de la culture scolaire, ils soutiennent généralement le processus de transmission de savoirs de l'école à la famille, en suscitant chez le parent un rôle de technicien ou de réceptacle d'informations de nature scolaire ou, dit autrement, d'agent de soutien de seconde ligne (Crozier et Davies, 2007; Doherty, 2000; Fleer et Williams-Kennedy, 2001). La pratique du travailleur social tend alors à se situer dans une perspective clinique, son action éducative et socioéducative visant à instrumenter le parent au plan scolaire et à compenser les carences éducatives associées aux difficultés scolaires de l'enfant (Larose, Lenoir, Couturier et Bédard, 2011sp).

Ce rôle, souvent adopté par le travailleur social dans le cadre de projets ou de programmes se réclamant d'une logique partenariale, ne concorde pas avec la position d'interface qu'il est traditionnellement appelé à occuper dans le cadre de sa pratique. En effet, son action éducative et socioéducative vise foncièrement à favoriser les liens sociaux et, dans cette logique, à soutenir la participation des parents en tant que membres et agents de construction de la culture. Traditionnellement, il soutient aussi l'articulation entre les objets culturels auxquels s'expose l'enfant lors de ses transitions entre plusieurs systèmes, tels que la famille, l'école et la communauté.

Une tension, de nature potentiellement identitaire, s'exprime alors pour le travailleur social. En effet, son agir professionnel ne concorde pas avec la fonction de «partenaire » qu'il est appelé à exercer dans le cadre de programmes à caractère partenarial, surtout en matière de soutien à la participation parentale. Afin de contribuer à une certaine réconciliation entre les « champs » au sein desquels il se positionne, nous proposons de définir son rôle dans une perspective de médiation culturelle.

\section{Le travailleur social comme médiateur culturel}

Devant le constat d'un écart entre la culture de la famille et celle de l'école et du maintien, voire du renforcement de la rupture que vit l'enfant lors de ses transitions sociales entre ces systèmes, les auteurs se réfèrent de plus en plus à la fonction d'interface exercée par un tiers intervenant, le plus souvent le psychologue scolaire (Bates, 2005; Guli, 2005; Pelco, Jacobson, Ries et Melka, 2000). L'action partenariale de ce dernier se constate surtout aux États-Unis, ce 
qui est aussi le cas du travailleur social. Qu'elle vise à soutenir les pratiques pédagogiques de l'enseignant (Sheridan et Welch, 1996) ou à faciliter l'adaptation sociale (Allen et Blackston, 2003; Warren, Bohanon-Edmonson, Turnbull, Sailor, Wickham, Griggs et Beech, 2006) de l'enfant à l'école, l'intervention vise à favoriser une certaine articulation entre les pratiques éducatives des parents et celles des personnels scolaires. Certains auteurs renvoient spécifiquement au rôle de médiateur ${ }^{7}$ culturel exercé par un ensemble de professionnels des métiers relationnels (psychologue, direction d'école, travailleur social) (Auerbach, 2009; Bryan et Henry, 2008; Hodges, 2009; Price-Mitchell, 2009). Au Québec, une telle fonction est principalement remplie par les psychoéducateurs. Elle est aussi associée aux agents de liaison, une profession émergente proche du travail social (Larose, Boulanger, Couturier, Bédard et Larivée, 2009; Couturier et al., 2010). Le travailleur social, qui jadis exerçait un tel rôle, est appelé à resituer sa pratique au cœur des systèmes école, famille et communauté (Larose, Couturier et Boulanger, 2007).

Dans une perspective socioculturelle, l'intervenant est en quelque sorte considéré comme un ethnologue qui procède à une lecture des cultures entre lesquelles circule l'enfant, dans le but d'en décoder les normes, les représentations, les pratiques sociales de référence ainsi que les savoirs de sens commun (Cairney, 1994, 1997). II cherche à créer des passerelles entre ces univers culturels, en favorisant, chez chacun des acteurs (les parents, l'enseignant, etc.), la prise de conscience et la reconnaissance des compétences de leurs tiers, compétences associées à la diversité des pratiques de nature éducative (Cairney, 2001; Carter, 2006; Fleer et William-Kennedy, 2001).

L'enseignant est appelé à tenir compte, dans la réalisation des activités en classe, des pratiques sociales des familles dont l'univers culturel diffère de ce qui est véhiculé à l'école (Samaras et Wilson, 1999). Parallèlement, les parents s'exposent à la culture scolaire et, dans une certaine mesure, ils s'y intègrent et y contribuent par le biais de leur participation sociale à l'école (Delgato-Gaitan, 1992; Cairney, 1994). Ils peuvent alors soutenir de manière optimale les apprentissages de l'enfant et ses transitions sociales.

L'intervenant doit non seulement favoriser cette forme d'intégration culturelle de la part de chaque catégorie d'acteurs, mais aussi faciliter le dialogue entre eux (Fleer et Williams-Kelly, 2001). Il doit créer des ponts entre l'école et la famille, en s'assurant que chaque participant adopte une position de mutualité et de réciprocité (Christenson, 2004). Les rôles éducatifs, les savoirs et les pratiques éducatives sont négociés par le biais d'interactions constructives et soutenues entre les acteurs, interactions médiées par un tiers (Moll, 1993; Price-Mitchell, 2009).

7. La notion de médiation n'est pas entendue au sens strict d'un rapport aux savoirs auquel renvoie le champ de la didactique, bien que notre analyse s'inscrive en partie dans cette perspective. Nous donnons à la médiation le sens que lui attribue Engeström $(1987,2001)$, soit une forme de « processus » ou de « dynamique » qui permet l'articulation entre plusieurs objets sémantiques et qui passe par l'usage d'outils symboliques et culturels (le langage, des pratiques, des écrits, des représentations sociales). Dans cette logique, les objets en question (des objets de savoir, qui se transcrivent notamment au plan des croyances et des attitudes) interagissent à la charnière entre plusieurs systèmes sociaux. La participation sociale y est considérée comme un «moyen » favorisant la médiation culturelle entre les objets auxquels s'expose l'individu au sein et à l'interface des systèmes qu'il fréquente. 
De manière plus large, cet intervenant crée une communauté de pratique, où les parents et les personnels scolaires co-construisent une culture de pratique (Valsiner, 2009). II est souhaitable, voire primordial d'y intégrer les membres de la communauté et les professionnels qui y interviennent et, plus largement, les « citoyens », dans le but de favoriser l'émergence et la consolidation d'un réseau de pratiques intégrées (Grigorenko, 2001). Nous inscrivant au cœur du champ des « activity theory » (Engeström, 1987, 2001), nous ne définissons pas cette culture comme un ensemble d'objets et de pratiques partagés de manière relativement uniforme, mais bien d'objets de savoir hétéroclites évoqués, construits, constitués et mobilisés dans l'action et l'interaction, par le biais de la co-participation sociale, et qui prennent ancrage au sein d'un environnement culturel (Bruni, Gherardi et Parolin, 2007).

Cette participation réciproque est associée à la co-construction ${ }^{8}$ de savoirs culturels et à leur articulation ainsi qu'à la réalisation d'apprentissages sociaux de la part de chaque acteur impliqué (Fleer, 2006). Il s'agit d'apprentissages situés contextuellement et fondés sur l'acquisition et l'appropriation à la fois sociale et individuelle de savoirs de nature culturelle et symbolique (Rogoff, 1997). Dans cette perspective, les parents, au même titre que les professionnels, sont considérés comme des apprenants qui conjointement réalisent des apprentissages sociaux et développent et s'approprient des compétences qui sont véhiculées à l'enfant par le biais d'un réseau de pratiques intégrées dont ils sont partie prenante (Grigorenko, 2001; Bruni, Gherardi et Parolin, 2007). Lorsqu'ils s'inscrivent dans le champ de l'intervention éducative et socioéducative, les écrits en travail social abordent les interactions et la participation du parent sous l'angle des apprentissages sociaux de ce dernier, bien que ce discours ne soit qu'au stade de l'émergence au Québec (Terrisse et Larose, 2003; Couturier, Larose et Bédard, 2009).

\section{ConClusion}

Considérant que la profession du travail social évolue dans le contexte de la mise en œuvre d'un partenariat entre l'école, la famille et la communauté et que les pratiques qui s'y inscrivent impliquent une articulation complexe de l'action éducative et socioéducative de diverses catégories de professionnels, il nous a semblé important de mettre en perspective le rôle de soutien à la participation parentale qu'est appelé à y jouer le travailleur social, à la lumière des pratiques qu'il exerce en termes de participation parentale, cette notion balisant fortement son agir professionnel. Sur cette base, nous avons pu relever la tension qu'implique le passage

8. Lorsque nous faisons référence à la dimension de négociation des rôles et de co-construction de pratiques et de savoirs, nous ne nous situons pas dans une logique strictement situationnelle associée à une approche conversationnelle ou constructionniste (Lyotard, 1979) où la composante « locale » prévaut et où les savoirs sont constamment renouvelés par un processus dit émergent. Dans notre perspective, les activités, qui se réalisent contextuellement et quotidiennement, comprennent, dans leur actualisation, une certaine part d'innovation ou, plus particulièrement, elles conduisent à l'émergence de savoirs. Ces derniers tendent toutefois à prendre ancrage et à se sédimenter pour constituer des objets culturels, du fait que les activités se réalisent sur une longue période de temps et qu'elles s'organisent autour de certains patrons d'action et d'éléments de régularité. Ce sont ces derniers qui caractérisent largement les pratiques socioculturelles. Dit autrement, les activités se déploient au gré de contextes variés et évolutifs mais comportent une dimension « stable » par leur ancrage dans une culture particulière. Dans la logique élaborée par Engeström $(1987,2001)$, il serait préférable à nos yeux de traiter d'un ensemble de cultures fusionnées (au sens dialectique du terme), les individus interagissant au cœur et à la charnière de systèmes sociaux interreliés. Pour cette lecture de l'écosystème ou de l'écosystémie, consulter Overton (1998) et Lerner (2006). 
d'une logique de soutien à la participation sociale des parents, considérés comme des « membres » d'un environnement social partageant des repères de nature socio-symbolique, à une autre où l'accent est mis sur un processus unilatéral de transmission d'informations de nature scolaire. La conception socioculturelle de la participation parentale que nous avons soulevée nous a permis d'identifier certaines zones de correspondance entre le rôle attribué au travailleur social et ce qui caractérise ses pratiques à ce chapitre. Ce faisant, nous avons proposé de définir le rôle exercé par le travailleur social au plan de la participation parentale dans une logique de médiation culturelle. Nous avons identifié trois grandes zones de pratiques potentielles, soit, d'une part, un travail de mobilisation des acteurs autour de la culture de leurs tiers (les amener à décoder cette culture et à la reconnaître), d'autre part, un exercice de liaison entre ces acteurs et, finalement, une action visant la construction sociale de savoirs et leur appropriation dans une logique d'apprentissage social.

Or, l'adoption d'une perspective socioculturelle suppose aussi de situer cette intervention éducative et socioéducative au cœur de la culture de pratique du travailleur social et, plus particulièrement, de tenir compte de ses propres référents socio-symboliques et culturels, soit de ses représentations sociales. Parce qu'elles influencent de manière marquante son action en matière de soutien à la participation parentale et la nature de son interaction avec le parent, les représentations sociales qu'il porte eu égard au rôle et aux compétences du parent s'avèrent particulièrement centrales. C'est en tant que membre d'une communauté de pratique et, plus largement, d'une culture particulière, qu'il adhère à ces représentations, qu'il se les approprie, qu'il les construit et les actualise par le biais de ses pratiques d'intervention. Son appartenance à une culture particulière, la position hiérarchique qu'il y occupe, son rôle et la nature de l'interaction qu'il y entretient avec des tiers constituent des objets complémentaires à l'analyse que nous avons développée dans le cadre de cet article. 
Dany Boulanger

Étudiant au doctorat en éducation

Université de Sherbrooke

Maîtrise en travail social Baccalauréat en psychologie

François Larose, Ph. D.

Professeur titulaire en enseignement au préscolaire et au primaire Université de Sherbrooke Doctorat en éducation.

\section{Serge J. Larivée, Ph.D.}

Professeur agrégé Département de psychopédagogie et d'andragogie Université de Montréal Doctorat en éducation

\section{Pauline Minier, Ph.D.}

Professeure titulaire Université du Québec à Chicoutimi Doctorat en éducation

\section{Yves Couturier, Ph.D.}

Professeur titulaire Service Social, Université de Sherbrooke Doctorat en sciences humaines appliquées

Jean-Claude Kalubi-Lukusa, Ph.D.

Professeur titulaire Département d'études sur l'adaptation scolaire et sociale

Université de Sherbrooke Doctorat en éducation Postdoctorat, adaptation scolaire et sociale

Valérie Cusson, DMD.

Étudiante à la maîtrise en sciences cliniques

Université de Sherbrooke Doctorat en médecine dentaire (DMD). 


\section{RÉFÉRENCES BIBLIOGRAPHIQUES}

Allen, S. J. et A. R. Blackston (2003). «Training preservice teachers in collaborative problem solving: An investigation of the impact on teacher and student behaviour change in real-world settings », School Psychology Quarterly, 18(1), p. 22-51.

Auerbach, S. (2009). «Walking the walk: portraits in leadership for family engagement in urban schools », The School Community Journal, 19(1), p. 9-31.

Bates, S. L. (2005). «Evidence-based family-school interventions with preschool children », School Psychology Quarterly, 20(4), p. 352-370.

Boethel, M. (2003). Diversity. School, Family and Community Connections. Annual synthesis 2003, Austin (TX), National Center for Family and Community Connections with Schools/Southwest Educational Development Laboratory.

Boudreault, P., J.-C. Kalubi et A. C. Moreau (2000). «Compétences des parents et évaluation du développement de leur enfant: de l'appropriation à l'autodétermination », Les Cahiers de l'Actif, 288291, p. $165-178$.

Boudreault, P., J.-C. Kalubi, L. Sorel, P. Beaupré et J.-M. Bouchard (1998). "Recherches sur l'appropriation des savoirs et des savoir-faire entre parents et intervenants », dans S. L. Éthier et J. Alary, Comprendre la famille, Québec, Presses de l'Université du Québec, p. 316-330.

Boulanger, D., F. Larose et Y. Couturier (2010). «La logique déficitaire en intervention sociale auprès des parents: les pratiques professionnelles et les représentations sociales », Nouvelles pratiques sociales, 23(1), p. 152-176.

Bronfenbrenner, U. (1979). The Ecology of Human Development. Experiments by Nature and Design, Cambridge, Harvard University Press.

Bronfenbrenner, U. et G. W. Evans (2000). «Developmental science in the 21st century: Emerging questions, theoretical models, research designs and empirical findings », Social Development, 9(1), p. 115-125.

Bronfenbrenner, U. (2005). Making Human Beings Human: Bioecological perspectives on human development, Thousand Oaks (CA), Sage.

Bruni, A., S. Gherardi et L. L. Parolin (2007). « Knowing in a system of fragmented knowledge », Mind, Culture, and Activity, 12(1-2), p. 83-102.

Bryan, J. et L. Henry (2008). « Strengths-based partnerships: A school-family-community partnership approach to empowering students », Professional School Counseling, 12(2), p. 149-156.

Cairney, T. H. (1994). «Family literacy: Moving towards new partnerships in education », Australian Journal of Language and Literacy, 17(4).

Cairney, T. H. (1997). « Acknowledging diversity in home literacy practices: moving towards partnership with parents », Early Child Development and Care, 127-128, p. 61-73.

Cairney, T. H. (2000). « Beyond the classroom walls: The rediscovery of the family and community as partners in education », Educational Review, 52, p. 163-174. 
Cairney, T. H. (2001). « Bridging home and school literacy: in search of transformative approaches to curriculum », Early Child Development and Care, 172(2), p. 153-172.

Carter, S. (2006). « Redefining literacy as a social practice », Journal of Basic Writing, 25(2), p. 94-125.

Christenson, S. L. (2004). "The family-school partnership: An opportunity to promote the learning competence of all students », School Psychology Review, 33(1), p. 83-104.

Christenson, S. L. et S. M. Sheridan (2001). School and families: Creating essential connections for learning, New York, Guilford Press.

Clénet, J. et C. Gérard (1994). Partenariat et alternance en éducation. Des pratiques à construire, Paris, L'Harmattan.

Couturier, Y. (2005). La collaboration entre travailleuses sociales et infirmières. Éléments d'une théorie de l'intervention interdisciplinaire, Paris, L'Harmattan.

Couturier, Y. et F. Larose (2006). «Transitions et médiations croisées en intervention éducative. Conditions et potentialités d'une recherche collaborative en milieu scolaire », Esprit critique. Revue électronique de sociologie, 8(1). [http://www.espritcritique.org.]

Couturier, Y., F. Larose et J. Bédard (2009). « Continuités et discontinuités de l'intervention éducative et de l'éducation socioéducative en milieu scolaire défavorisé », Les sciences de l'éducation pour l'ère nouvelle, 42(4), p. 93-116.

Couturier, Y., L. Potvin, M. Lortie, F. Etheridge, I. Lantagne, J. Lacasse-Bédard et J. Bédard (2010). « Modélisation d'un métier du lien social dans le cadre d'un programme d'intervention nutritionnelle en contexte scolaire défavorisé », Nouvelles pratiques sociales, 22(2), p. 145-158.

Crozier, G. et J. Davies (2007). « Hard to reach parents or hard to reach schools? A discussion of homeschool relations, with particular reference to Bangladeshi and Pakistani parents », British Educational Research Journal, 33(3), p. 295-313.

Delgado-Gaitan, C. (1992). "School matters in the Mexican-American home: socializing children to education », American Educational Research Journal, 29(3), p. 495-513.

Deniger, M.-A. et G. Roy (2002). Enquête auprès des membres votants des conseils d'établissement, Groupe d'analyse politique de l'éducation (CRIRES), Québec, Université Laval, Faculté des sciences de l'éducation.

Department of Education (2006). Goals 2000: Educate America Act. [http://www.state.nj.us/education/educators/goals2000.htm.]

Department of Education (2007). No Child Left Behind Act. [http://www.ed.gov/policy/elsec/leg/nclb/buildingonresults.pdf.]

Deslandes, R. et Bertrand, R. (2004). « Motivation des parents à participer au suivi scolaire de leur enfant au primaire », Revue des sciences de l'éducation, 30(2).

[http://www.erudit.org/revue/RSE/2004/v30/n2/012675ar.html.]

Deslandes, R. et A. Lemieux (2005). " The place of family and community within schools in Québec », dans D. B. Hiatt-Michael (dir.), Promising Practices in Family Involvement in Schooling Across the Continents, Connecticut, Information Age Publishing, p. 93-112. 
Doherty, W. J. (2000). « 1999 NCFR Presidential Address. Family science and family citizenship: Toward a model of community partnership with families », Family Relations, 49, p. 319-325.

Eldridge, D. (2001). « Parent involvement: It's worth the effort », Young Children, 56(4), p. 65-69.

Engeström, Y. (1987). Learning by expanding: An activity-theoretical approach to developmental research, Helsinki, Orienta-Konsultit Oy.

Engeström, Y. (2001). « Expansive learning at work: Toward an activity theoretical reconceptualization », Journal of Education and Work, 14(1), p. 133-156.

Epstein, J. L. (1995). « School/family/community partnerships: Caring for the children we share », Phi Delta Kapan, 76, p. 701-711.

Epstein, J. L. (2005). «A case study of the Partnership Schools Comprehensive School Reform (CSR) model », Elementary School Journal, 106(2), p. 151-170.

Fan, X. T. et M. Chen (2001). «Parental involvement and students' academic achievement: A metaanalysis », Educational Psychology Review, 13, p. 1-22.

Fleer, M. (2006). «Troubling cultural fault lines: some indigenous australian families' perspectives on the landscape of early childhood education », Mind, Culture, and Activity, 13(3), p. 191-204.

Fleer, M. et D. Williams-Kennedy (2001). Building bridges: Literacy development in young indigenous children, Canberra, Australian Early Childhood Association Inc.

Franklin, A. et A. Sloper (2005). « Participation of disabled children and young people in decision making within social services departments: A survey of current and recent activities in England », British Journal of Social Work, 36, p. 723-741.

Gauvain, M. (2005). « Sociocultural contexts of learning », dans A. E. Maynard et M. I. Martini (dir.), Learning in cultural context: Family, peers, and school, New York, Springer, p. 11-40.

Geoghegan, M. et F. Powell (2006). « Community development, partnership governance and dilemmas of professionalization: profiling and assessing the case of Ireland », British Journal of Social Work, 36, p. 845-861.

Gouvernement du Québec (2000). Consolider la collaboration entre le milieu scolaire et le milieu communautaire: une mesure clé pour la réussite éducative des jeunes, Québec, Ministère de l'Éducation du Québec.

Gouvernement du Québec (2002a). Les services éducatifs complémentaires : essentiels à la réussite, Québec, Ministère de l'Éducation du Québec.

Gouvernement du Québec (2002b). Programme de recherche sur la persévérance et la réussite scolaires, Québec, Ministère de l'Éducation du Québec.

Gouvernement du Québec (2003a). Stratégie de mise en œuvre 2003-2006 de l'Entente de complémentarité des services entre le réseau de la santé et des services sociaux et le réseau de l'éducation, Québec, Ministère de la Santé et des Services sociaux et Ministère de l'Éducation, du Loisir et du Sport.

Gouvernement du Québec (2003b). Programme national de santé publique 2003-2012, Québec, Ministère de la Santé et des Services sociaux. 
Gouvernement du Québec (2003c). Le plaisir de réussir se construit avec mon entourage. Cadre de référence, Québec, Ministère de l'Éducation, du Loisir et du Sport.

[http://www.mels.gouv.qc.ca/dfga/politique/fecre/pdf/cadre_fecre.pdf.]

Gouvernement du Québec (2003d). Les difficultés d'apprentissage à l'école. Cadre de référence pour guider l'intervention, Québec, Ministère de l'Éducation, du Loisir et du Sport.

Gouvernement du Québec (2005). L'école communautaire. Un carrefour pour la réussite des jeunes et le développement de la communauté, Québec, Équipe de travail sur le développement de l'école communautaire.

Gouvernement du Québec (2006a). Unis dans l'action. Plan d'action interministériel en toxicomanie 20062011, Québec, Ministère de la Santé et des Services sociaux.

Gouvernement du Québec (2006b). Investir pour l'avenir. Plan d'action gouvernemental de promotion des saines habitudes de vie et de prévention des problèmes reliés au poids 2006-2012, Québec, Ministère de la Santé et des Services sociaux.

Gouvernement du Québec (2007). Orientations relatives aux standards d'accès, de continuité, de qualité, d'efficacité et d'efficience. Programme-services Jeunes en difficulté 2007-2012, Québec, Ministère de la Santé et des Services sociaux.

Gouvernement du Québec (2008). Programme national de santé publique 2003-2012. Mise à jour 2008, Québec, Ministère de la Santé et des Services sociaux.

Gouvernement du Québec (2009a). Programme de soutien à l'école montréalaise, Québec, Ministère de l'Éducation, du Loisir et du Sport.

Gouvernement du Québec. (2009b). L'école, j'y tiens. Tous ensemble pour la réussite scolaire. Québec, Ministère de l'Éducation, du Loisir et du Sport.

Grigorenko, E. L. (2001). « Arguing for the concept of developmental niche », dans E. L. Grigorenko et R. J. Sternberg (dir.), Family Environment and Intellectual Functioning: A life-span perspective, Mahwah (NJ), Lawrence Erlbaum Associates, p. 23-47.

Guli, L. A. (2005). "Evidence-based parent consultation with school-related outcomes », School Psychology Quarterly, 20(4), p. 455-472.

Henderson, A. T. et K. L. Mapp (2002). A New Wave of Evidence: The impact of school, family, and community connections on student achievement, Austin (TX), Southwest Educational Development Laboratory. [http://www.sedl.org/pubs/catalog/items/fam33.html.]

Henrich, C. C. (2006). "Context in action: implications for the study of children and adolescents », Journal of Clinical Psychology, 62(9), p. 1083-1096.

Hodges, S. (2009). «Mediation and counseling services: A viable partnership », Journal of College Student Psychotherapy, 23, p. 30-39.

Hoover-Dempsey, K. V. et H. M. Sandler (1995). «Parental involvement in children's education: Why does it make a difference? », Teachers College Record, 97(2), p. 310-331.

Jordan, C., E. Orozco et A. Averett (2001). Emerging Issues in School, Family and Community Connections, Austin (TX), National Center for Family and Community Connections with Schools/ Southwest Educational Development Laboratory. 
Larivée, S., J.-C. Kalubi et B. Terrisse (2006). «La collaboration école-famille-communauté en contexte d'inclusion: entre obstacles, risques et facteurs de réussite », Revue des sciences de l'éducation, 32(15), p. 525-543.

Larose, F., D. Boulanger, Y. Couturier, J. Bédard et S. J. Larivée (2009). « As representações sociais como analisador da emergência de uma nova profissão em educação: o caso dos agentes de desenvolvimento no Québec », Revista da FAEEBA - Educação e Contemporaneidade, 18(32), p. 129-146.

Larose, F., Y. Couturier et D. Boulanger (2007). «Conditions et enjeux de l'actualisation de l'interdisciplinarité professionnelle pour les formations initiales à l'enseignement et à l'exercice du travail social au Québec », Les dossiers des sciences de l'éducation, 17, p. 77-93.

Larose, F., Y. Lenoir, Y. Couturier et J. Bédard (2011sp). « L'essor de l'interdisciplinarité professionnelle au temps des réformes à la faveur de l'émergence d'une zone d'intégration des pratiques d'intervention éducatives et socio-éducatives », Revue des sciences de l'éducation, vol. XXXV, n 3 .

Larose, F., B. Terrisse, Y. Lenoir et J. Bédard (2004). « Approche écosystémique et fondements de l'intervention éducative précoce en milieux socio-économiquement faibles. Les conditions de la résilience scolaire », Brock Education, 13(2), p. 56-80.

Lawson, M. A. (2003). « School-family relations in context », Urban Education, 39(1), p. 77-134.

Lenoir, Y., F. Larose, C. Deaudelin, J.-C. Kalubi et G.-R. Roy (2002). «L'intervention éducative: clarifications conceptuelles et enjeux sociaux. Pour une reconceptualisation des pratiques d'intervention en enseignement et en formation à l'enseignement », Esprit critique. Revue électronique de sociologie, 4(4).

Lerner, R. M. (2006). «Resilience as an attribute of the developmental system. Comments on the papers of professors Masten \& Wachs », Annals of the New York Academy of Sciences, 1094, p. 40-51.

Lewin, K. (1935). «Psycho-sociological problems of a minority group », Character and Personnality, 3(3), p. $175-187$.

Lewin, K. (1943). «Psychology and the process of group living », Journal of Social Psychology, 17(1), p. 113-131.

Lewin, K. et P. Grabbe (1945). « Conduct, knowledge, and acceptance of new values », Journal of Social Issues, 1(3), p. 53-64.

Lewin, K., R. Lippitt et R. K. White (1939). «Patterns of aggressive behaviour in experimentally created "social climates" », Journal of Social Psychology, 19(2), p. 271-299.

Lott, B. (2001). «Low-income parents and the public schools », Journal of Social Issues, 57(2), p. 247259.

Lupiani, J. L. (2004). Parental Role Construction, Parent Sense of Efficacy, and Perceptions of Teacher Invitations as Factors Influencing Parent Involvement, thèse de doctorat, Fordham University, New York.

Lyotard, J.-F. (1979). La condition post moderne : rapport sur le savoir, Paris, Éditions de Minuit.

Magnusson, D. et H. Stattin (1998). "Person-context interaction theories », dans W. Damon et R. M. Lerner (dir.), Handbook of Child Psychology, 6 édition, New York, John Wiley and Sons, p. 685-760. 
Mead, M. (1940). «Social change and cultural surrogates », Journal of Educational Sociology, 14(2), p. 92-109.

Mead, M. (1944). «Women's social position », Journal of Educational Sociology, 17(8), p. 453-462.

Mead, M. (1945a). " Observations of emotions and thinking in the living room », Journal of Social Issues, 1(2), p. 43-46.

Mead, M. (1945b). «Group living as a part of intergroup education workshops », Journal of Educational Sociology, 18(9), p. 526-534.

Mead, M. (1983). "Cultural discontinuities and personality transformation », Journal of Social Issues, 39(4), p. 161-177.

Mead, M. (2001). « The school in American culture », Society, 39(1), p. 54-62.

Mills, C. et T. Gale (2007). «Researching social inequalities in education: Toward a bourdieuian methodology », International Journal of Qualitative Studies in Education, 20(4), p. 433-447.

Moll, L. (1993). «Community-mediated educational practices », communication présentée à l'American Educational Research Association Annual Conference, Atlanta (GA), 12-16 avril.

Nichols, S. (2007). «Parental involvement in supporting children with learning difficulties », dans Louden et al. (dir.), Mapping the Territory. Primary students with learning difficulties: literacy and numeracy, Canberra, Department of Education, Training and Youth Affairs.

Ogbu, J. U. (1992). «Adaptation to minority status and impact on school success », Theory Into Practice, vol. XXXI, nº 4, p. 287-295.

Overton, W. F. (1998). «Developmental psychology: philosophy, concepts, and methodology », dans W. Damon et R. M. Lerner (dir.), Handbook of Child Psychology, $6^{\mathrm{e}}$ édition, New York, John Wiley and Sons, p. 107-188.

Pelco, L. E., L. Jacobson, R. R. Ries et S. Melka (2000). « Perspectives and practices in family-school partnerships: A national survey of school psychologists », School Psychology Review, 29(2), p. 235250.

Pelligrini, A. D., L. Galda et D. Charak (1997). «Bridges between home and school literacy: Social bases for early school literacy », Early Child Development and Care, 127-128, p. 99-109.

Powell, D. R. (2001). « Visions and realities of achieving partnership: Parent-teacher relationships at the turn of the century », dans M. von Cranach, W. Doise et G. Mugny (dir.), Social Representations and the Social Bases of Knowledge, Lewiston (NY), Hogrefe and Huber, p. 333-357.

Price-Mitchell, M. (2009). «Boundary dynamics: Implications for building parent-school parnterships », The School Community Journal, 19(2), p. 9-26.

Proulx, J.-P. (2009). Le système éducatif du Québec. De la maternelle à l'Université, Montréal, Chenelière Éducation.

Rogoff, B. (1997). «Evaluating development in the process of participation », dans E. Amsel et A. Renning, Change and Development: Issues of Theory, Method, and Application, Hillsdale (NJ), Erlbaum, p. 265-284. 
Roose, R., A. Mottart, N. Dejonckheere, C. Van Nijnatten et M. De Bie (2009). « Participatory social work and report writing », Child and Family Social Work, 14(3), p. 322-330.

Roth, W. M., K. Tobin, R. Elmesky, C. Carambo, Y.-M. McKnight et J. Beers (2004). "Re/making identities in the praxis of urban schooling: A cultural historical perspective », Mind, Culture, and Activity, 11(1), p. 48-69.

Samaras, A. P. et J. C. Wilson (1999). « Am I invited? Perspectives of family involvement with technology in inner-city schools », Urban Education, 34, p. 499-530.

Sanders, M. G. et K. C. Lewis (2004). «Partnerships at an urban high school: Meeting the parent involvement requirements of No Child Left Behind », E-Journal of Teaching and Learning in Diverse Settings, 2(1), p. 78-98.

Sanders, M. G. et B. S. Simon (2002). « A comparison of program development at elementary, middle, and high schools in the National Network of Partnership Schools », The School Community Journal, 12(1), p. 7-27.

Sheldon, S. B. (2003). «Linking school-family-community partnerships in urban elementary schools to student achievement on state tests », Urban Review, 35(2), p. 149-165.

Sheldon, S. B. (2005). « Testing a equation structural model of partnership program implementation and parent involvement », Elementary School Journal, 106(2), p. 171-187.

Sheridan, S. M. et M. Welch (1996). «Is consultation effective? », Remedial and Special Education, 17(6).

Shommer-Aikins, M. (2004). «Explaining the epistemological belief system: Introducing the embedded systemic model and coordinated research approach », Educational Psychologist, 39(1), p. 19-29.

Simon, B. S. (2004). « High school outreach and family involvement », Social Psychology of Education, 7 , p. $185-209$.

Sing, C. C. et M. S. Khine (2006). "An analysis of interaction and participation patterns in online community », Educational Technology and Society, 9(1), p. 250-261.

Solas, J. (1996). "The limits of empowerment in human service work », Australian Journal of Social Issues, 31(2), p. 147-156.

Sontag, J. C. (1996). «Toward a comprehensive theoretical framework for disability research: Bronfenbrenner revisited », The Journal of Special Education, 30(3), p. 319-344.

Souto-Manning, M. et K. Swick (2006). «Teachers' beliefs about parent involvement: Rethinking our family involvement paradigm », Early Childhood Education Journal, 34(2), p. 187-193.

Terrisse, B. et F. Larose (2003). « La formation des intervenants socio-éducatifs auprès de l'enfant et de la famille : une nouvelle profession, de nouvelles compétences », Revue internationale de l'éducation familiale, 7(1), p. 5-7.

Terrisse, B., F. Larose et Y. Couturier (2003). «Quelles sont les compétences attendues pour assurer la professionnalité dans l'intervention socio-éducative auprès du jeune enfant et de sa famille? », Revue internationale de l'éducation familiale, 7(1), p. 11-31. 
Tudge, J. R. H., J. Gray et D. Hogan (1997). «Ecological perspectives in human development: A comparison of Gibson and Bronfenbrenner », dans J. Tudge, M. Shanahan et J. Valsiner (dir.), Comparisons in Human Development: Understanding time and context, New York, Cambridge University Press, p. 72-105.

Valsiner, J. (2003a). «Beyond social representations: A theory of enablement », Papers on Social Representations, 12, p. 7.1-7.16.

Valsiner, J. (2003b). «Culture and its transfer: Way of creating general knowledge through the study of cultural particulars », Online Readings in Psychology and Culture, 2(11).

Valsiner, J. (2009). « Cultural psychology todays: Innovations and oversights », Culture and Psychology, 15(1), p. 5-39.

Valtonen, K. (2002). «Social work with immigrants and refugees: Developing a participation-based framework for anti-oppressive practice - Part 2 », British Association of Social Workers, 32, p. 113120.

Van Velsor, P. et G. I. Orozco (2007). « Involving low-income parents in the schools: Communitycentric strategies for school counsellors », Professional School Counseling, 11, p. 17-24.

Vincent, C. et Tomlinson, S. (1997). « Home-school relationships: 'The swarming of disciplinary mechanisms'? », British Educational Research Journal, 23(3), p. 361-377.

Warren, J. S., H. M. Bohanon-Edmonson, A. P. Turnbull, W. Sailor, D. Wickham, P. Griggs et S. E. Beech (2006). « School-wide positive behavior support: Addressing behavior problems that impede student learning », Educational Psychology Review, 18, p. 187-198.

Wong, W. C. (2001). «Co-constructing the personal space-time totality: Listening to the dialogue of Vigotsky, Lewin, Bronfenbrenner, and Stern », Journal for the Theory and Social Behaviour, 31(4), p. 365-382. 